\title{
Synthesis and characterization of silica aerogel-based nanocomposites with carbon fibers and carbon nanotubes in hybrid system
}

\author{
Agnieszka Ślosarczyk ${ }^{1}$
}

Received: 6 January 2017 / Accepted: 7 July 2017 / Published online: 24 July 2017

(C) The Author(s) 2017. This article is an open access publication

\begin{abstract}
In presented research the silica aerogel nanocomposites with carbon microfibers and nanofibers, as well as carbon microfibers/carbon nanotubes hybrid system were investigated. It was shown, that an important element of tailoring the properties of silica aerogel nanocomposite was the right choice of the type and the length of the carbon material. In case of carbon nanofibers with length of $20 \mathrm{~nm}$, the applied amount of fibers was too low to keep the silica aerogel frame in monolith and resulted in obtaining the nanocomposite in form of granulate. Only longer fibers with length of $700 \mu \mathrm{m}$ gave the monolith structure of silica aerogel nanocomposite. As a result of hybrid reinforcement, which enables reduction of cracking on micro and nano level, a monolith nanocomposite was received, which had very good structural parameters and low contraction during drying. All the received nanocomposites were characterized by good thermal resistance and all applied carbon materials did not deteriorate the high thermal resistance of silica aerogel.
\end{abstract}

Graphical Abstract
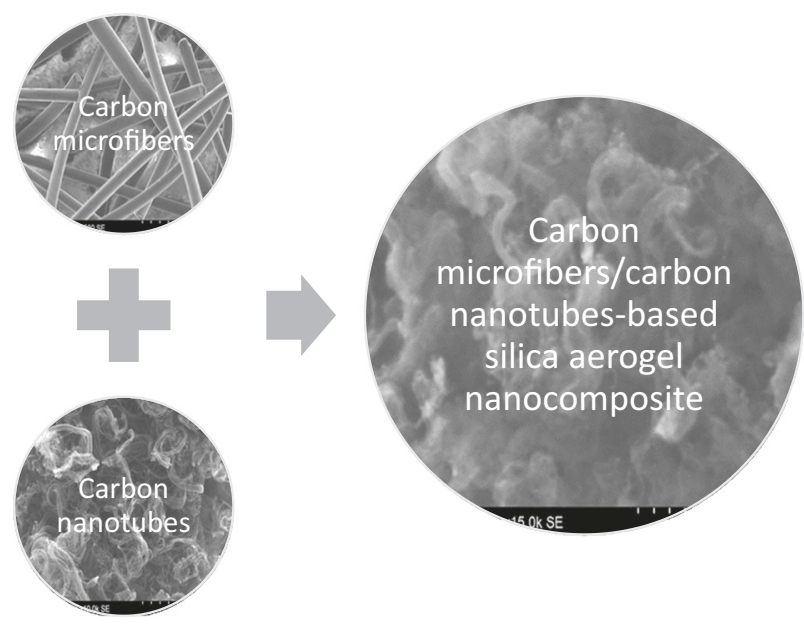

Keywords Silica aerogel nanocomposites $\cdot$ Ambient pressure drying $\cdot$ Carbon microfibres and nanofibers $\cdot$ Carbon nanotubes $\cdot$ Chemical modification

\section{Introduction}

Besides unique properties like low density, developed specific surface, high porosity, low thermal conductivity and transparency, which guarantee them application in many branches of industry [1-4], the silica aerogels have also some disadvantages, from which, the most important from the applicable point of view is the brittleness. One of the way of improving this drawback is the reinforcement of silica aerogel frame by short randomly distributed fibers [5] 
or by usage of fibers mats (http://www.aerogel.com, http:// www.cabotcorp.com). There are many types of fibers and nanofibers witch can be applied to strengthening the silica frame, e.g., ceramic [6-8], glass [9], aramid [10], polypropylene [11], polyaniline [12], cotton [13], and carbonbased [14-17]. Relatively little attention has been paid to the silica aerogel nanocomposites with carbon-based materials. Carbon fibers and nanofibers, characterized by good mechanical parameters and relatively low density, are resistant to temperature above $600^{\circ} \mathrm{C}$ and are biocompatible $[18,19]$, which enables their application as components of nanocomposites with silica aerogel not only in the technical industry, but also in biomaterials areas. Moreover, those fibers can be modified in the surface, which enables chemical bonding of surface function groups on the fiber surface with hydroxyl groups present on the surface of the silica aerogel, and results in obtaining a nanocomposite with better structural parameters $[16,17]$.

In opposite to the previous works $[16,17]$ in presented research the influence of higher amounts of carbon microfibers on the structural parameters of silica aerogel-based nanocomposites were tested. Moreover, the influence of carbon nanofibers and for the first time, the hybrid systemcarbon microfibers/carbon nanotubes on the structure and properties of silica aerogel matrix were verified. The physico-chemical characterization of received silica aerogel nanocomposites were carried out by means of Brunauer-Emmett-Teller (BET), termogravimetric (TG), and scanning electron microscopy (SEM) analysys.

\section{Experimental part}

\subsection{Materials}

In presented research the three types of carbon materials were used, namely: pitch-based carbon microfibers (DONACARBO), vapor grown carbon nanofibers VGCFs (ShowaDenco) [20], and polyhedral carbon nanotubes (ALDRICH). Carbon microfibers with diameter of $13 \mu \mathrm{m}$ and length of $700 \mu \mathrm{m}$ are characterized by very good mechanical parameters (tensile strength $750 \mathrm{MPa}$, elastic modulus $40 \mathrm{GPa}$ ), high thermal stability up to $750{ }^{\circ} \mathrm{C}$, density of $1.64 \mathrm{~g} / \mathrm{cm}^{3}$, carbon content over $99 \%$ and relatively high degree of structure order. The distance between graphite planes defined in the Bragg equation equals about $0.353 \mathrm{~nm}$. Naturally those fibers are characterized by low degree of specific surface development, around $4 \mathrm{~m}^{2} / \mathrm{g}$, and high hydrophobicity. Thus, the fibers applied in the aerogel composites, were earlier chemically modified in concentrated nitric acid, in higher temperature in order to oxidize the fiber surface and give it a hydrophilic character, which was described in detail in previous articles and the monograph [16, 17, 21]. Second material used in the research are the carbon nanofibers VGCF received from gas phase of the hydrocarbons. The fibers are 10-20 nm long with diameter of $150 \mathrm{~nm}$ and specific structure consisting of inner empty fibril surrounded by graphite layers that are perpendicular to the axis of the fiber. This characteristic fiber structure results in very good mechanical parameters of the fibers, which also have good thermal and electric parameters. Those fibers have the density of $2 \mathrm{~g} / \mathrm{cm}^{3}$ and specific surface of $13 \mathrm{~m}^{2} / \mathrm{g}$, as well as a much better degree of structure order than fibers from carbon pitch (the distance between the planes is $0.340 \mathrm{~nm}$ ). Those fibers, before they were applied in aerogel composites, also underwent chemical modification in concentrated nitric acid, in higher temperature in order to oxidize the fiber surface and give it a hydrophilic character. As the third carbon material the polyhedral carbon nanotubes with length equaled 0.5-200 $\mu \mathrm{m}$, outer diameter $7-15 \mathrm{~nm}$, inner diameter 3-6 nm, density $2.1 \mathrm{~g} / \mathrm{cm}^{3}$ and carbon content over $95 \%$ were applied. Carbon nanofibers were received with chemical vapor deposition CVD with the use of catalyst Co-Mo in high temperature process of gas $\mathrm{CO}$ disproportionation by pressure of $1-10$ at [22].

\section{Methods}

To characterize the physical and chemical properties of the received nanocomposites the following measuring techniques were applied. Surface area and pore volume of aerogel composites were estimated based on adsorption isotherms in low-temperature nitrogen sorption in the temperature of 77 $\mathrm{K}$ using the equation of BET isotherm and analyzer ASAP 2010 (Micrometrics). Thermo-gravimetric analysis of aerogels was performed in argon atmosphere by means of NETSCH apparatus, type STA 409C. During measurements the following parameters were used: flow rate of inert gas $10 \mathrm{ml} / \mathrm{min}$, speed of sample heating $5^{\circ} \mathrm{C} / \mathrm{min}$, and temperature range $30-1000^{\circ} \mathrm{C}$. The microstructure of the nanocomposites were investigated by SEM with TESCAN3VEGA microscope. The thermal conductivity of silica aerogel and its nanocomposites with carbon materials was measured by means of hot disc thermal analyzer LaserComp FOX314. The equipment enables the testing of flat samples with dimensions of $305 \times 305 \times 100 \mathrm{~mm}$ in the range of thermal expansion coefficient between 0.01 and $0.2 \mathrm{~W} /$ $(\mathrm{m} \bullet \mathrm{K})$ with the accuracy of $1 \%$, maximum temperature of hot plate equals $75^{\circ} \mathrm{C}$, while minimum temperature of cold plate $-20^{\circ} \mathrm{C}$. The analyzer measures the thermal conductivity which is defines as the amount of heat per unite time and unit area that can be conducted through a plate of unit thickness according to Fourier-Biota correlation $q=$ $-\lambda(d T / d x)$. 

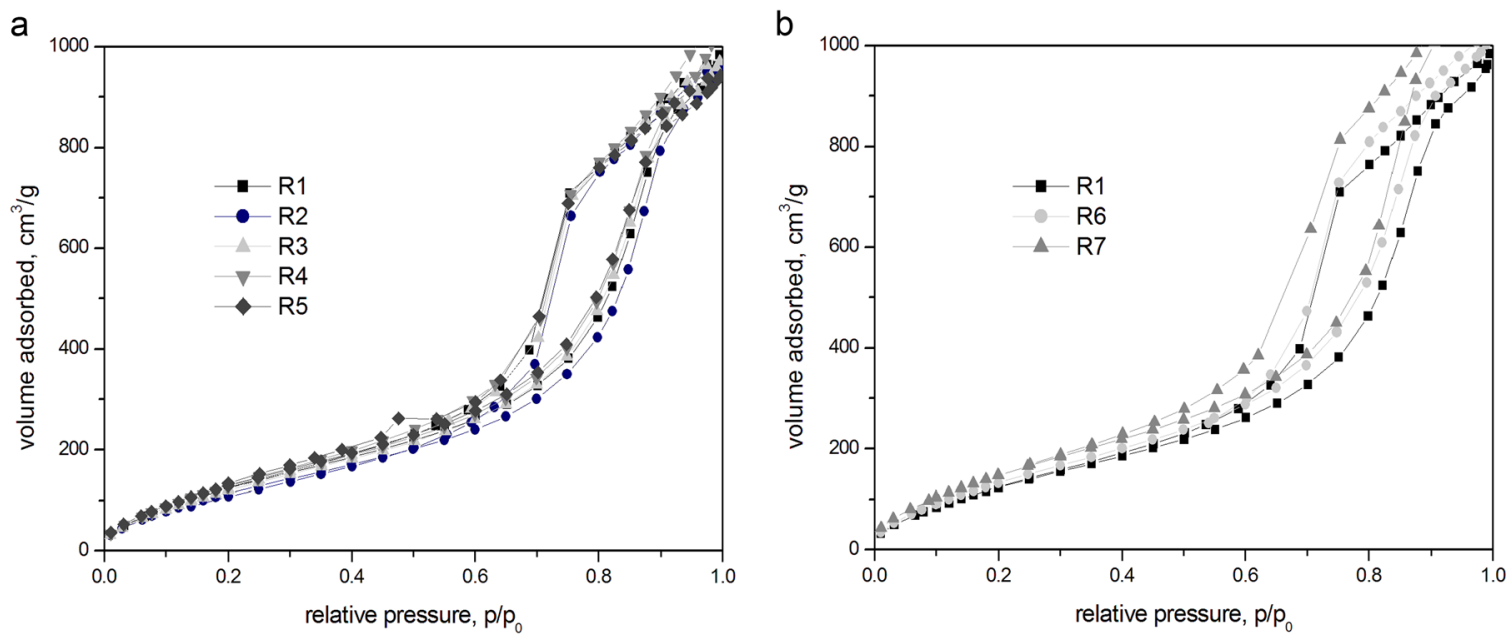

Fig. 1 a, b Adsorption/desorption isotherms for silica aerogel nanocomposites (R1-pure silica aerogel, R2-with 15 vol\% CFs, R3-with 10 vol\% CFs, R4-with 15 vol\% CFs and CnTs, R5-with 10 vol\% CFs and CnTs, R6-with 15 vol\% CnFs, R7-with 10 vol\% CnFs)

\subsection{Synthesis of silica aerogel nanocomposites}

Silica aerogel was received from TEOS in a two-stage sol-gel synthesis, with the use of $\mathrm{NH}_{4} \mathrm{OH}$ catalyst. In the first stage TEOS was mixed with ethanol, and then $0.1 \mathrm{M}$ solution of hydrochloric acid was added for the initial hydrolysis of the mixture. Next, an $\mathrm{NH}_{4} \mathrm{OH}$ reaction catalyst was added (concentration $1.5 \mathrm{M}$ ) so that a quick gelation took place. It is necessary by synthesis of nanocomposite with carbon fibers. The catalyst concentration must be adjusted in such a way, that the gelation time is shorter than the process of the fiber and nanofiber sedimentation in the reaction mixture. It results in good distribution of fibrous materials in the whole volume of the nanocomposite. Carbon microfibers and nanofibers were added to the TEOS solution in ethanol before gelation stage, in the amount of 10 and $15 \mathrm{vol} \%$ and mixed in a magnetic stirrer for about $15 \mathrm{~min}$, so that a suspension of fibers was received. Additionally, carbon microfibers and nanotubes in a hybrid system were used. Part of the carbon microfibers was replaced with $30 \mathrm{mg}$ of polyhedral carbon nanotubes. When the good dispersion of carbon materials in the TEOS solution was obtained, the reaction catalyst was added and a quick gelation took place. In the next stage, the received silica gels, with and without carbon materials underwent the process of ageing in ethanol for 7 days, and next their surface was modified in a mixture of TMCS/n-hexane in the volume ratio $1 / 10$ in $50{ }^{\circ} \mathrm{C}$ for $48 \mathrm{~h}$. In the last stage, the nanocomposites were dried in the air.

\section{Results and discussion}

Figure 1 shows the adsorption/desorption $\mathrm{N}_{2}$ isotherms of silica aerogel based nanocomposites with carbon microfibers, carbon nanofibers and hybrid system-carbon microfibers/carbon nanotubes. All tested nanocomposites exhibited the typical shape of isotherms corresponds to mesoporous materials. According to classification of porous materials made by de Boer in 1958, the shape of the desorption hysteresis loop indicates the pores structure with both cylindrical capillary pores open at both ends and cylindrical pores closed at one end with a narrow neck at the other, like an "ink-bottle". The average pores diameters for all materials are similar and vary between 9.9 and $11.4 \mathrm{~nm}$. Some differences appear in micropores volume. In case of silica aerogel with carbon nanotubes and nanofibers, due to the higher specific area of those materials in comparison with carbon microfibers, the increase of total micropores volume was observed. This also influenced positively the specific area of silica aerogel with carbon nanotubes and nanofibers.

Table 1 presents structural characteristics of the received silica aerogel and nanocomposites with carbon fibers and nanotubes. In case of pure aerogel-after the material was dried-a homogeneous structure was not obtained; during drying the sample cracked and an aerogel granulate was received with density around $0.194 \mathrm{~g} / \mathrm{cm}^{3}$ and specific surface about $640 \mathrm{~m}^{2} / \mathrm{g}$. Contraction in volume equaled over $65 \%$. In case of formula R6 and R7, reinforced with 15 and $10 \%$ of carbon nanofiber, also an aerogel granulate was received, with density similar to that of pure aerogel, respectively, 0.212 and $0.195 \mathrm{~g} / \mathrm{cm}^{3}$. Research proved that carbon nanofibers applied in the amount of 15 and $10 \%$ of volume were too short to obtain a homogeneous structure of nanocomposite, despite the fact that they slightly lowered the contraction of the sample during drying. Such amount of nanofibers resulted also in decreasing the specific surface area of the nanocomposite, which equaled 566.2 and 598 $\mathrm{m}^{2} / \mathrm{g}$, respectively for R6 and R7 formula. Only application 
Table 1 Structural characteristics of the received silica aerogel and its nanocomposites with carbon fibres, nanofibers, and nanotubes

\begin{tabular}{llllllll}
\hline Sample $^{\mathbf{a}}$ & R1 & R2 & R3 & R4 & R5 & R6 & R7 \\
\hline Density, g/cm & & 0.194 & 0.156 & 0.164 & 0.153 & 0.158 & 0.212 \\
Surface area by BET, m ${ }^{2} / \mathrm{g}$ & 640.4 & 502.9 & 550.0 & 537.9 & 558.1 & 566.2 \\
Average pore diameter, nm & 10.9 & 11.4 & 10.7 & 10.6 & 10.8 & 9.9 & 589.8 \\
Micropore volume, $\mathrm{cm}^{3} / \mathrm{g}$ & 1.744 & 1.436 & 1.476 & 1.438 & 1.512 & 1.406 \\
Volume shrinkage, \% & 65 & 48.1 & 47.7 & 46.4 & 31.9 & 64.7 \\
Form & Granulate & Monolith & Monolith & Monolith & Monolith & Granulate \\
\hline
\end{tabular}

${ }^{a}$ R1-pure silica aerogel, R2-with 15 vol\% CFs, R3- with 10 vol\% CFs, R4- with 15 vol\% CFs and CnTs, R5 with 10 vol\% CFs and CnTs, R6- with 15 vol\% CnFs, R7- with 10 vol\% CnFs

of much longer carbon microfibers resulted in obtaining a homogeneous structure of the silica xerogel. Presented research proved that both, 10 and 15 vol\% addition of carbon fibers lowered the density of the aerogel nanocomposite, the density for formulas R2 and R3 equaled respectively 0.156 and $0.164 \mathrm{~g} / \mathrm{cm}^{3}$. Structural analysis with BET method proved, that carbon microfibers as well as carbon nanofibers did not disturb the characteristic mesoporous structure of the silica aerogel, although the specific surfaces and average diameters of pores of the received nanocomposite were slightly smaller, in comparison with values obtained for pure aerogel granulate. However, a positive feature was the lowering the contraction of samples during drying, which equaled respectively for formulas R2 and R3 48.1 and $47.7 \%$.

Very interesting results were obtained for application of hybrid reinforcement in formulas R4 and R5, where part of the carbon microfibers was replaced with polyhedral carbon nanotubes. It turned out that such small amounts of nanotubes with very good mechanical parameters resulted in further lowering of the composite density, values gained for R4 and R5 equaled respectively 0.153 and $0.158 \mathrm{~g} / \mathrm{cm}^{3}$. Presence of such a small amount of nanotubes, characterized by much bigger specific surface than carbon microfibers, caused the specific surface of the nanocomposite to grow, while keeping the same pore layout, the diameters of which equaled around $10.8 \mathrm{~nm}$. Moreover, those nanocomposites were characterized by lower contraction during drying. The best results were gained for formula R5 with $10 \mathrm{vol} \%$ content of carbon microfibers and polyhedral carbon nanotubes, where the contraction during drying was below $32 \%$, which was over two times lower than that of pure silica aerogel. The silica aerogel is brittle in nature, it means that such matrix has good resistance to compressive stress, but is fragile to tensile and bending stresses. In case of silica aerogel dried in ambient pressure conditions an additional internal stresses from the spring-back effect occur. It makes the silica aerogel more fragile and easily-cracked. From the other side it is well known that the interfacial bonding between fibers and matrix depends on many factors, from which the most important are: the mechanical interlocking of fibers in matrix and the chemical and physical bonding between the fibers and matrix [23, 24]. Further, the mechanical interlocking depends on the aspect ratio of the fibers (length to diameter factor $1 / \mathrm{d}$ ), the shape and texture of fibers, as well as the amount of fibers per matrix volume. Whereas the adhesion can be enhanced by roughness of fibers and the chemical interaction between fibers surface and matrix. The use of carbon fibers and nanocarbons with very good $1 / \mathrm{d}$ ratio, which can be easily modified by oxidation seems to be a very good solution. In previous research [17] it was shown that the oxidative treatment of carbon fibers surface resulted in better structural parameters of silica aerogel nanocomposite and lowered its contraction during drying due to the chemical reaction between carboxylic groups localized on the modified fibers and the hydroxyl groups on silica aerogel frame. However, in presented results, it was shown that the surface modification and high amount of nanofibers are insufficient to obtain the silica aerogel nanocomposite in monolith form. The nanofibers, with quite good $1 / \mathrm{d}$ ratio, are too short to maintain the structure of nanocomposite in monolith and to prevent the microcracking of the silica aerogel. Only both, the longer carbon microfibers which enables reduction of cracking on micro level and the carbon nanotubes preventing nanocracking brought the expected results. A s a result of hybrid reinforcement, the best structural parameters and lowest contraction during drying were achieved. All the received nanocomposites were characterized by good thermal resistance and they did not deteriorate the high thermal resistance of silica aerogel. Figure 2 presents thermogravimetric curves for pure silica granulate and nanocomposites with carbon microfibers, nanofibers and nanotubes. Research proved, that almost all the nanocomposites were characterized by high structure resistance up to temperatures of $350^{\circ} \mathrm{C}$, only in case of formula R2 with $15 \%$ addition of carbon microfibers a $2 \%$ mass deficit was observed in this temperature. This proves that all the nanocomposites were characterized by high hydrophobicity of their structure, and the applied modification in a mixture of TMCS/n-hexane was effective. The slight decrease of mass up to $300^{\circ} \mathrm{C}$ can be the result of evaporation 

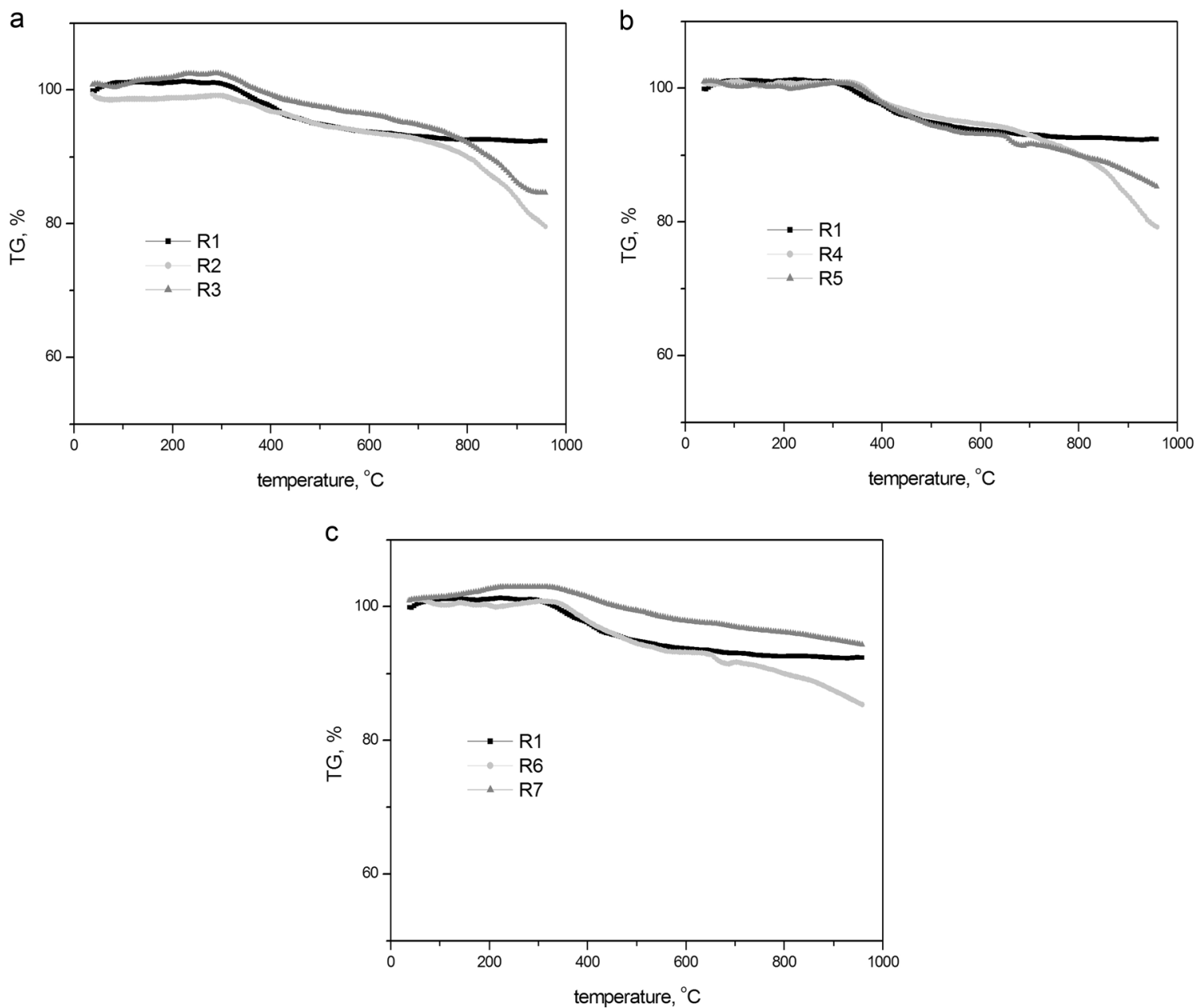

Fig. 2 a, b, c TG curves of carbon fibers-based silica aerogels synthesized from TEOS precursor in ambient pressure drying (R1-pure silica aerogel, R2-with 15 vol\% CFs, R3-with 10 vol\% CFs, R4-with
15 vol\% CFs and CnTs, R5-with 10 vol\% CFs and CnTs, R6-with 15 vol\% CnFs, R7-with 10 vol\% CnFs)

The higher amounts of nanofibers per volume unit of silica aerogel were also well dispersed in the silica aerogel matrix, but functioned only locally in micro regions bridging the micro cracks in $5 \mu \mathrm{m}$ length (Fig. 3d). As consequence the carbon nanofibers disturb the creation of monolith structure of nanocomposite which decomposed to granulate.

The obtained silica aerogel nanocomposite were also characterized by good thermal conductivity. On the Fig. 4 the dependences of thermal expansion coefficient in relation to the temperature for the carbon fibers based silica aerogel and the silica aerogel with the carbon microfibers/nanotubes system in the amount of $10 \mathrm{vol} \%$ were presented. It was shown that in the range of temperature from 10 to $40^{\circ} \mathrm{C}$ the thermal expansion coefficient for both samples equaled about $0.026-0.028 \pm 0.002 \mathrm{~W} /(\mathrm{m} \bullet \mathrm{K})$. However, above the temperature of $40^{\circ} \mathrm{C}$, the increase of temperature resulted in the increase of thermal expansion coefficient, up to $0.036 \pm$ $0.002 \mathrm{~W} /(\mathrm{m} \bullet \mathrm{K})$ at $62.5^{\circ} \mathrm{C}$. The increasing trend in thermal 

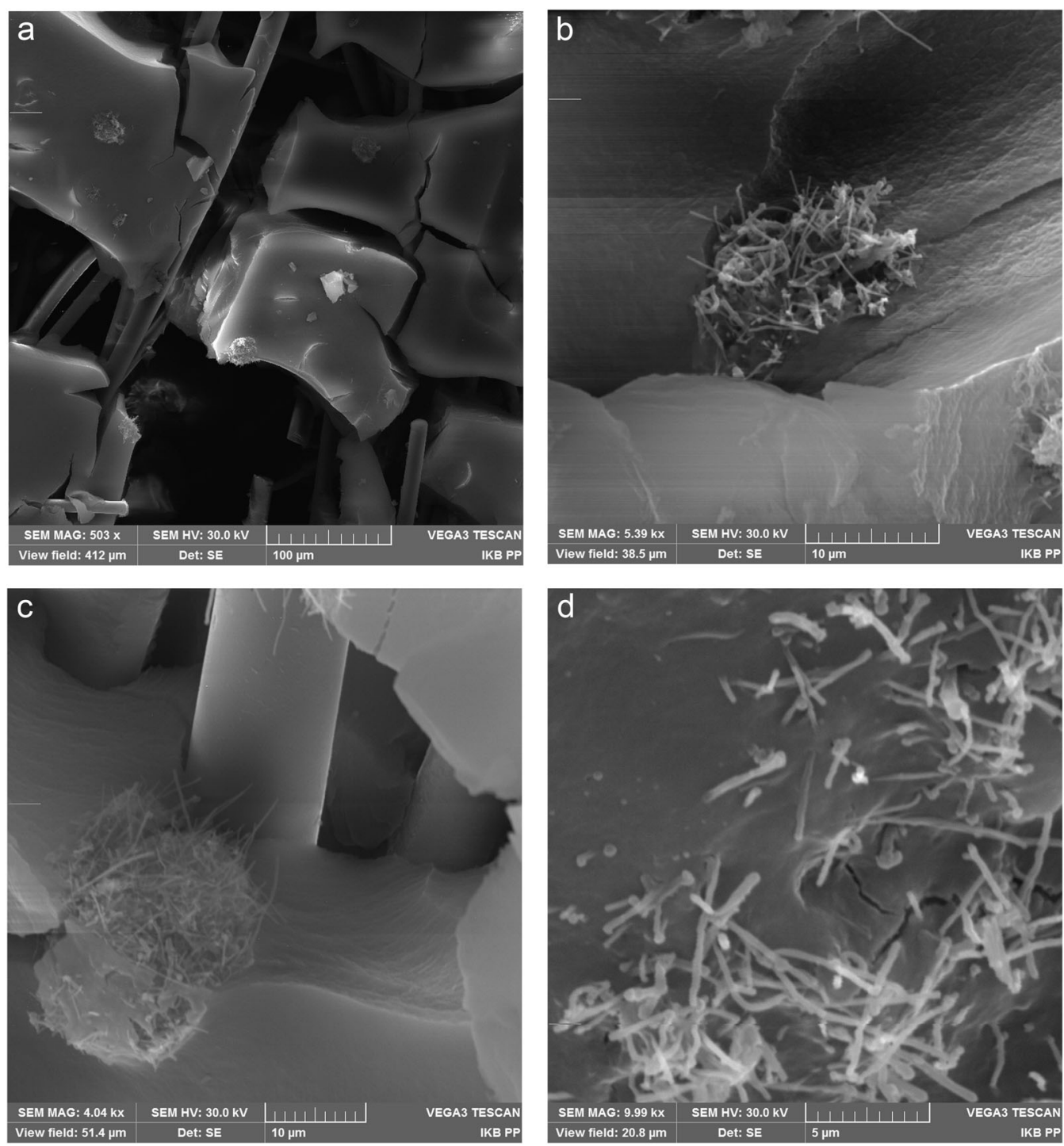

Fig. 3 a SEM picture of carbon microfibers-based silica aerogel. b SEM picture of carbon nanotubes in silica aerogel. c SEM picture of carbon microfibers/carbon nanotubes in silica aerogel. d SEM picture of carbon nanofibers-based silica aerogel

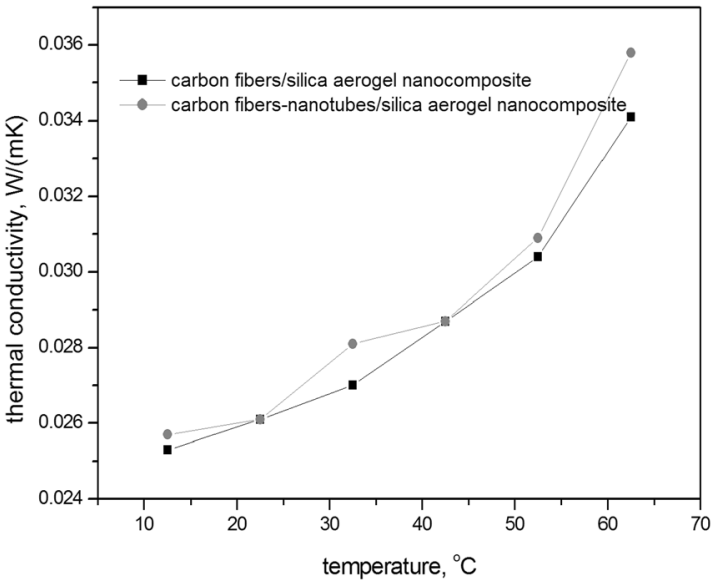

Fig. 4 Thermal conductivity in relation to the temperature for carbon microfibers and carbon microfibers/carbon nanotubes silica aerogel nanocomposites conductivity with respect to temperature was noticed also by others researchers for fibers-based silica aerogel nanocomposites [27, 28]. This increase is connected with the densification of mesoporous structure of silica aerogel, as well as the increasing thermal radiation of such nanomaterials in higher temperatures.

\section{Conclusions}

The presented research showed that an important element of tailoring the properties of silica aerogel nanocomposite was the right choice of the type and the length of the carbon material. In case of carbon nanofibers, such big amounts like 10 and 15 vol\% were insufficient to keep the silica aerogel frame in monolith and resulted in obtaining the 
nanocomposite in form of granulate. Only longer fibers with length of $700 \mu \mathrm{m}$ gave the monolith structure of silica aerogel nanocomposite. However, the best nanocomposite was obtained when both, the longer carbon microfibers and carbon nanotubes were used as silica aerogel reinforcement. In that case the cracking was reduced on the micro- and nano- level, which resulted in obtaining a monolith nanocomposite with very good structural parameters and much smaller contraction during drying, in comparison with carbon microfibers used separately. Due to thermal conductivity at the level of $0.026-0.028 \mathrm{~W} /(\mathrm{m} \bullet \mathrm{K})$ at room temperature such material can be applied as isolation material, especially as high temperature isolation. Moreover, the bigger amounts of carbon fibers and nanofibers, and their combination with carbon nanotubes, enable the shaping other properties of this material, e.g., the conductivity. With bigger amounts of carbon fibers and nanofibers in silica aerogel matrix, the percolation threshold is crossed, which makes possible to obtain a material with conductive properties, and potentially increase the application of this nanocomposite e.g., as electrode materials.

\section{Compliance with ethical standards}

Conflict of interest The authors declare that they have no competing interests.

Open Access This article is distributed under the terms of the Creative Commons Attribution 4.0 International License (http://crea tivecommons.org/licenses/by/4.0/), which permits unrestricted use, distribution, and reproduction in any medium, provided you give appropriate credit to the original author(s) and the source, provide a link to the Creative Commons license, and indicate if changes were made.

\section{References}

1. Aegerter MA (2011) Aerogels handbook, Springer, New York

2. Hrubesh LW (1998) J Non-Cryst Solids 225:335-342

3. Akimov YK (2003) Instrum Exp Tech 46:5-19

4. Cuce E, Cuce PM, Wood CJ, Riffat SB (2014) Renew Sust Energ Rev 34:273-299
5. Maleki H, Durãres L, Portugal A (2014) J Non-Cryst Solids 385:55-74

6. Paramente K, Milstein F (1998) J Non-Cryst Solids 223:179-189

7. Yang X, Sun Y, Shi D (2012) J Non-Cryst Solids 358:519-524

8. Yang X, Sun Y, Shi D, Liu J (2011) Mater Sci Eng A 528:4830-4836

9. Kim C-Y, Lee J-K, Kim B-I (2008) Colloids Surf A 313314:179-182

10. Li Z, Gong L, Cheng X, He S, Li C, Zhang H (2016) Mater Des 99:329-355

11. Zhang Z, Shen J, Ni X, Wu G, Zhou B, Yang M, Gu X, Qian M, Wu Y (2006) J Macrom Sci A 43:1663-1670

12. Boday DJ, Muriithi B, Stover RJ, Loy DA (2012) J Non-Cryst Solids 358:1575-1580

13. Razaei E, Moghaddas J (2016) Adv Mat Lett 7:296-301

14. Meador MA, Vivod S, McCorkle L, Quade D, Sullivan RM, Ghosn LJ, Clark N, Capadonna A (2008) J Mat Chem 18:1843-1852

15. Li L, Yalcin B, Nguyen B, Meador MA, Cakmak M (2009) Appl Mat Interfaces 11:2491-2501

16. Ślosarczyk A, Strauchmann W, Ziółkowski P, Jakubowska P (2015) J Non-Cryst Solids 416:1-3

17. Ślosarczyk A, Barełkowski M, Niemier Sz, Jakubowska P (2015) J Sol-Gel Sci Technol 76:227-232

18. Burchell TD (1999) Carbon materials for advanced technologies. Elsevier, Amsterdam

19. Chung DDL (1994) Carbon fiber composites. Butterworth-Heinemann, Newton

20. Endo M, Kim YA, Hayaski T, Nishimura K, Matusita T, Miyashita K, Dresselhaus M (2001) Carbon N Y 39:1287-1297

21. Ślosarczyk A (2013) Cement composites modified with selected carbon materials. LAP LAMBERT Academic Publishing, Saarbrücken, Germany

22. Patel K (2009) Nanomaterials controlled synthesis and properties. Materials Matters, 4:1-30, Sigma-Aldrich Corporation, Milwaukee, USA

23. Pathak AK, Borach M, Gupta A, Yokozeki T, Dhakate SR (2016) Comp Sci Tech 135:28-38

24. Zhang H, Zhang Z, Breidt C (2004) Comp Sci Tech 64:2021-2029

25. Li Z, Cheng X, He S, Shi X, Gong L, Zhang H (2016) Comp Part A $84: 316-325$

26. Li Z, Gong L, Cheng X, He S, Li C, Zhang H (2016) Mater Design 99:349-355

27. Wei TW, Lu SY, Chang YC (2009) J Phys Chem C 113:7424-7428

28. Tang X, Sun A, Chu Ch, Yu M, Ma S, Cheng Y, Guo J, Xu G (2017) Mater Design 115:415-421 